\title{
A high oxidative stress index predicts endothelial dysfunction in young male smokers
}

\author{
Karahan $\mathrm{O}^{1}$, Manduz $\mathrm{S}^{2}$, Bektasoglu $\mathrm{G}^{3}$, Zorlu $\mathrm{A}^{4}$, Turkdogan $\mathrm{KA}^{5}$, Bozok $\mathrm{S}^{6}$ \\ Medical School of Dicle University, Department of Cardiovascular Surgery, Diyarbakir, Turkey. \\ oguzk2002@gmail.com
}

\begin{abstract}
Experimental studies have shown that smoking was related to endothelial dysfunction via oxidative stress. However, the degree of oxidative stress to be associated with endothelial dysfunction is unknown. Oxidative stress index (OSI) might be a useful and easy way of determining the endothelial dysfunction. Hence, we aimed to evaluate the relationship between OSI and flow mediated dilatation (FMD) in smoking healthy male volunteers. Eighty smoking healthy male volunteers were enrolled in the study. Participants were classified as having normal and abnormal FMD response. In an univariate analysis; systolic and diastolic blood pressures, Creactive protein (CRP), low-density lipoprotein cholesterol, OSI and lipid peroxidation (LPO) levels were predictive for abnormal FMD response. In a multivariable logistic regression analysis with forward stepwise method, OSI (OR: 3.194, 95\% Cl: 1.710-5.966, p<0.001) and CRP (OR: 2.082, 95\% Cl: 1.101-3.939, p 0.024) were found to be independent parameters for predicting abnormal FMD response in young male smokers. The optimal cut-off value of OSI for detecting abnormal FMD response was found to be $>3.35$, with $100 \%$ sensitivity and $84.1 \%$ specificity. We have shown that critical endothelial dysfunction can easily be detected by OSI in individuals, at risk for developing coronary artery disease, such as smokers (Tab. 3, Fig. 3, Ref. 30). Text in PDF www.elis.sk. Key words: high oxidative stress index, endothelial dysfunction, young male smokers, systolic blood pressure, diastolic blood pressure, C-reactive protein, low-density lipoprotein, cholesterol, lipid peroxidation, coronary artery disease.
\end{abstract}

Endothelial dysfunction, oxidative stress and lipid peroxidation plays an important role in atherosclerosis (1-7). Experimental data have shown that smoking induced oxidative stress impairs endothelial dysfunction (8-9). However, in most of the clinical trials regarding smoking induced pathophysiology, endothelial dysfunction and oxidative stress was assessed separately.

Flow mediated dilatation (FMD), a novel technique for detecting endothelial dysfunction, is frequently used in recent years for detecting the degree of endothelial dysfunction (10). This was also true for oxidative stress index (OSI) in detecting oxidative stress status. FMD is a noninvasive test and probably because of this, has become one of the most frequently used techniques for detecting endothelial dysfunction in clinical trials. However, it is time consuming, expensive, requires experienced sonographer and specialized probes, which is not always available in routine clinical practice. However, OSI can easily be evaluated from peripheral

${ }^{1}$ Medical School of Dicle University, Department of Cardiovascular Surgery, Diyarbakir, Turkey, ${ }^{2}$ Medical School of Cumhuriyet University, Department of Cardiovascular Surgery, Sivas, Turkey, ${ }^{3}$ Karsiyaka State Hospital, Department of Cardiology, Izmir, Turkey, ${ }^{4}$ Muş Bulanik State Hospital, Department of Cardiology, Muş, Turkey, ${ }^{5}$ Bezmialem Vakif University, Faculty of Medicine, Department of Emergency Medicine, Istanbul, Turkey, and 'Rize Education and Research Hospital, Department of Cardiovascular Surgery, Rize, Turkey

Address for correspondence: O. Karahan, MD, Medical School of Dicle University, Department of Cardiovascular Surgery, Diyarbakir, Turkey. Phone: +90.5063929320 , Fax: +903462581503 blood samples. Along with its ease of use, we think OSI can replace FMD in order to detect endothelial dysfunction in patients at risk for developing coronary artery disease and future cardiovascular events. To the best of our knowledge, such relationship has not been evaluated so far. Based on this idea, we evaluated the relationship between FMD and OSI.

\section{Materials and methods}

\section{Study population}

Eighty healthy male volunteers, who have been smoking for at least 5 years, were enrolled into the study. None had clinically ascertainable recent acute or chronic disease. The study protocol was approved by the local ethics committee. Written informed consent was obtained from participants.

\section{Blood sample collection}

Blood samples were obtained following an overnight fasting. Samples were withdrawn from the cubital vein of the nondominant arm into blood tubes and stored at $-80{ }^{\circ} \mathrm{C}$. The serum was separated from the cells by centrifugation at 3,000 rpm for $10 \mathrm{~min}$ and then analyzed.

\section{Measurement of lipid peroxidation (LPO)}

The measurement of lipid peroxidation (thiobarbituric acid reactive substances) was performed according to the method described by Yagi (11). After an extraction with buthanol, serum 
samples were boiled with thiobarbituric acid and the absorbance of the pink colour at the upper phase of the liquid was measured at $532 \mathrm{~nm}$. The results were defined using a standard solution of 1,1,3,3-tetraethoxypropane and were expressed as nanomoles of malondialdehyde (MDA) per millilitre serum (nmol/ml MDA).

\section{Measurement of total oxidant status (TOS)}

The total oxidant status of plasma was measured using a novel automated colorimetric measurement method for TOS developed by Erel (12). The assay is based on the oxidation of ferrous ion to ferric ion in the presence of various oxidant species in acidic medium and the measurement of the ferric ion by xylenol orange. The ferric ion makes a coloured complex with xylenol orange in an acidic medium. The colour intensity, which can be measured spectrophotometrically, is related to the total amount of oxidant molecules present in the sample. The assay is calibrated with hydrogen peroxide and the results are expressed in terms of micromolar hydrogen peroxide equivalent per litre $\left(\mu \mathrm{mol} \mathrm{H}_{2} \mathrm{O}_{2} \mathrm{Eq} / \mathrm{l}\right)$.

\section{Measurement of total antioxidant capacity (TAC)}

Total antioxidant capacity of plasma was measured using a novel automated colorimetric measurement method for TAC developed by Erel (13). In this method, hydroxyl radical is produced by the Fenton reaction and it reacts with the colourless substrate O-dianisidine to produce the dianisyl radical, which is bright yellowishbrown in colour. After addition of a plasma sample, the oxidative reactions initiated by the hydroxyl radicals present in the reaction are suppressed by the antioxidant components of the plasma, preventing the colour change and thereby providing an effective measurement of TAC. The assay results were expressed as mmol Trolox Eq/l.

\section{Determination of oxidative stress index (OSI)}

The ratio of TOS to TAC was accepted as the OSI. For calculation of OSI value following formula was used: OSI (arbitrary unit $)=\operatorname{TOS}\left(\mu \mathrm{mol} \mathrm{H}_{2} \mathrm{O}_{2} \mathrm{Eq} / \mathrm{l}\right) /$ TAC (mmol Trolox Eq/l) (14).

\section{Evaluation of flow mediated dilatation}

All participants were evaluated in the same morning with an overnight fasting after obtaining blood sample from the contrala- teral arm. Evaluations were made at room temperature $\left(\sim 25^{\circ}\right)$ by a single sonographer, who was blinded to the clinical and laboratory results of the study population. Linear array transducer with a frequency of $10 \mathrm{MHz}$ (Vivid 4 - Cardiovascular Ultrasound System, 10L transducer) was used to acquire images. The electrocardiogram was monitored continuously. A rrace of the dominant arm's brachial artery was evaluated above antecubital fossa. The best place for brachial artery imaging was marked by a pencil. After measuring the basal diameter of the brachial artery, the cuff of a sphygmomanometer was placed above antecubital fossa. Cuff was inflated at least $50 \mathrm{mmHg}$ above systolic blood pressure and waited for 5 minutes to induce ischemia at the brachial artery. After the cuff was deflated, lumen diameter was noted 1 min later to assess FMD. All measurements were done in the end-diastole (the beginning of the R on ECG) from endothelial to endothelial surface along a line perpendicular to the artery's long axis. FMD was expressed as the percentage change in vessel diameter during reactive hyperemia (10). Although a universally accepted threshold for defining a normal vs. abnormal FMD response has not been proposed, currently available evidence indicates that the ideal cut-off value may be around five percent (15-19). Based on the previous studies, a FMD value $<5 \%$ and $\geq 5 \%$ was accepted as abnormal and normal FMD response.

\section{Statistical analysis}

Continuous variables were expressed as the mean \pm standard deviation or medians (range) in the presence of abnormal distribution, categorical data as numbers and percentages. SPSS 15.0 (SPSS, Inc., Chicago, Illinois) was used to perform statistical procedures. Independent variables were compared via Independent sample's $t$ test. The Mann-Whitney U test was used to test parametric data without binomial distribution. Multivariable logistic regression analysis was used to evaluate independent variables for detecting abnormal FMD response. Receiver operator characteristic (ROC) curve analysis was performed to identify the optimal cut off point of OSI (at which sensitivity and specificity would be maximal) for the prediction of abnormal FMD response. The area under the curve (AUC) was calculated for detecting the accuracy of the test. The $\mathrm{p}$ value $<0.05$ was accepted as statistically significant.

Tab. 1. Baseline characteristics of groups.

\begin{tabular}{lcc}
\hline & $\begin{array}{c}\text { Normal flow mediated } \\
\text { dilatation }(\geq 5 \%)(\mathrm{n}=69)\end{array}$ & $\begin{array}{c}\text { Abnormal flow mediated } \\
\text { dilatation }(<5 \%)(\mathrm{n}=11)\end{array}$ \\
\hline Age (years) & $30 \pm 3$ & $31 \pm 3$ \\
Systolic blood pressure (mmHg) & $118 \pm 10$ & $129 \pm 15$ \\
Diastolic blood pressure (mmHg) & $72 \pm 6$ & $81 \pm 13$ \\
Fasting glucose (mg/dl) & $115 \pm 28$ & $124 \pm 10$ \\
Triglyceride (mg/dl) & $107 \pm 28$ & 0.372 \\
LDL (mg/dl) & $136 \pm 55$ & 0.009 \\
HDL (mg/dl) & $32 \pm 5$ & 0.058 \\
Total cholesterol (mg/dl) & $158 \pm 39$ & 0.098 \\
Creatinine (mg/dl) & $0.9 \pm 0,2$ & $31 \pm 3$ \\
Hemoglobin (gr/dl) & $14.5 \pm 1.3$ & 0.020 \\
C-reactive protein (mg/l) & $2.9 \pm 1.9(0-8)$ & 0.432 \\
Oxidative Stress Index & 0.270 \\
Lipid Peroxidation (nmol/ml MDA) & 0.647 & $0.9 \pm 0,1$ \\
\hline
\end{tabular}

LDL - Low-density lipoprotein cholesterol, HDL - High-density lipoprotein cholesterol 


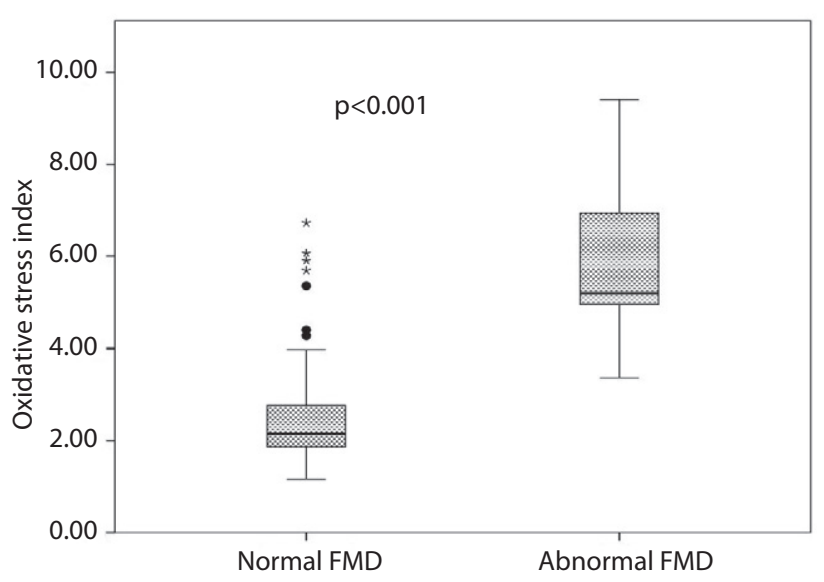

Fig. 1. Comparison of oxidative stress index levels between two groups.

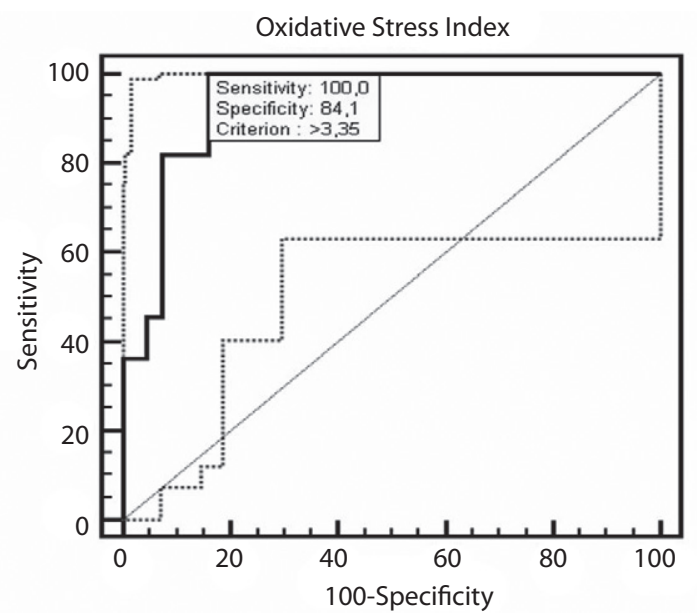

Fig. 2. ROC curves of oxidative stress index for detecting abnormal flow mediated dilatation response.

\section{Results}

The mean age of the study population was $30 \pm 3$. The mean systolic and diastolic blood pressure of the study population was $120 \pm 11$ and $73 \pm 8$, respectively. Four participants' blood pressures were in the hypertensive range at time of evaluation, however follow-up blood pressures of these participants were found to be normal. The mean OSI level of the study population was $3.0 \pm 1.7$ (range, 1.1-9.4), mean LPO level was 2.4 \pm 0.7 (range 1.27-4.07). Eleven participants (14\%) out of 80 have abnormal FMD response and 69 participants $(76 \%$ ) have normal FMD response.

Baseline characteristics of groups are listed in the Table 1. Fasting glucose, lipid parameters (except for LDL), hemoglobin and creatinine levels were similar between the groups. Individuals with abnormal FMD response had significantly higher systolic and diastolic blood pressures ( $\mathrm{p}=0.016$ and $\mathrm{p}=0.009$, respectively). C-reactive protein and LDL levels were found to be significantly higher in individuals with abnormal FMD response (p: 0.004 and

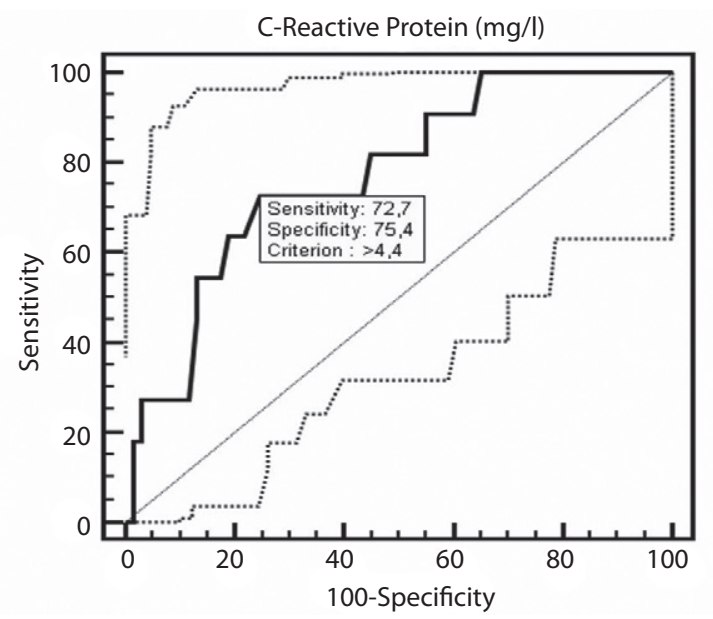

Fig. 3. ROC curves of C-reactive protein for detecting abnormal flow mediated dilatation response.

p: 0.020, respectively). Oxidative stress index and LPO levels were also found to be significantly higher in individuals with abnormal FMD ( $p<0.001$, for each). The comparison of median OSI levels between the two groups is shown in the Figure 1.

Univariate predictors of abnormal FMD response are listed in the Table 2. Of the 6 variables that were significant in the univariate model, only two remained so in the multivariate logistic regression model and OSI (OR: 3.194, 95\% CI: 1.710-5.966, $\mathrm{p}<0.001$ ) remained the strongest independent predictor of abnormal FMD response, followed by CRP (OR: 2.082, 95\% CI: 1.101-3.939, p: 0.024) (Tab. 3).

The optimal cut-off value of OSI for detecting abnormal FMD response was found to be $>3.35$, with $100 \%$ sensitivity and $84.1 \%$ specificity (AUC $0.941,95 \%$ CI 0.889 to 0.992 ) (Fig. 2). Also, the optimal cut-off value of CRP for detecting abnormal FMD response was found to be $>4.4 \mathrm{mg} / \mathrm{l}$, with $72.7 \%$ sensitivity and $75.4 \%$ specificity (AUC $0.774,95 \%$ CI 0,667 to 0.860 ) (Fig. 3 ).

Tab. 2. Univariate predictors of abnormal flow mediated dilatation response.

\begin{tabular}{lccc}
\hline & $\mathrm{p}$ & OR & $(95 \% \mathrm{CI})$ \\
\hline Oxidative Stress Index & $<0.001$ & 3.000 & $1.735-5.188$ \\
Lipid Peroxidation (nmol/ml MDA) & $<0.001$ & 6.753 & $2.431-18.756$ \\
C-reactive protein (mg/l) & 0.006 & 1.753 & $1.178-2.609$ \\
Systolic blood pressure (mmHg) & 0.010 & 1.086 & $1.020-1.156$ \\
Diastolic blood pressure (mmHg) & 0.003 & 1.153 & $1.048-1.268$ \\
LDL (mg/dl) & 0.077 & 1.011 & $0.999-1.024$ \\
\hline
\end{tabular}

All the variables from Table 1 were examined and only those significant at $p<0.1$ level are shown. CI - Confidence interval; OR - Odds ratio

Tab. 3. Multivariate predictors of abnormal flow mediated dilatation response.

\begin{tabular}{lccc}
\hline & $\mathrm{p}$ & $\mathrm{OR}$ & $(95 \% \mathrm{CI})$ \\
\hline Oxidative Stress Index & $<0.001$ & 3.194 & $1.710-5.966$ \\
C-reactive protein $(\mathrm{mg} / \mathrm{l})$ & 0.024 & 2.082 & $1.101-3.939$ \\
\hline
\end{tabular}

Multivariate logistic regression model including all the variables from Table 2. CI Confidence interval; OR - Odds ratio 
$721-725$

\section{Discussion}

It is already known that endothelial dysfunction assessment via FMD was clearly associated with atherosclerosis and its complications (20-22). Smoking, the major risk factor for coronary artery disease was also known to be associated with $\operatorname{FMD}(23,24)$. Moreover, smoking cessation also causes improvement in endothelial dysfunction (24). Oxidative stress takes play an important role early in atherosclerotic process and several studies has shown an association between FMD and oxidative stress in different patient populations (25-28). Su et al have shown that superoxide dismutase was positively correlated to FMD in patients with diabetes and pre-diabetes. Cangemi et al have also shown this relationship in patients with metabolic syndrome. Edirisinghe et al demonstrated that cigarette smoke induced oxidative stress impaired fluid shear stress mediated signalling in endothelial cells in an experimental study (8). Another experimental study has also shown the role of superoxide anion in cigarette-smoke induced endothelial dysfunction (29). Kato et al have shown a relationship between plasma 8-Isoprostane levels and FMD in smokers. They also showed the acute effects of passive smoking on FMD and 8-Isoprostane levels (30). However, none of the clinical studies currently available in literature has shown the direct relationship between OSI and FMD. To our knowledge, also the degree of oxidative stress to be associated with endothelial dysfunction in smokers was not known. Our study has shown a clear relationship between OSI and FMD. An OSI level above 3.35 clearly designates individuals with severe endothelial dysfunction.

Our study has one major limitation. Relatively small size of the study population and volunteers with abnormal FMD response may have influenced the results. As it could be appreciated, due to the relatively young age of the study population, it was so hard to find healthy persons with such degree of endothelial dysfunction (FMD $<5 \%$ ).

\section{Conclusion}

Atherosclerosis and its complications is the leading cause of mortality in worldwide. This makes novel techniques, for detecting atherosclerosis and its implications early in the disease process, invaluable. By its ease of use, we think OSI might replace FMD in order to detect endothelial dysfunction, individuals at risk for developing coronary artery disease and future cardiovascular events.

\section{References}

1. Ross R. Atherosclerosis - an inflammatory disease. N Engl J Med 1999; 340: $115-126$.

2. Antoniades C, Shirodaria C, Crabtree M, Rinze R, Alp N, Cunnington C, Diesch J, Tousoulis D, Stefanadis C, Leeson P, Ratnatunga C, Pillai R, Channon KM. Altered plasma versus vascular biopterins in human atherosclerosis reveal relationships between endothelial nitric oxide synthase coupling, endothelial function, and inflammation. Circulation 2007; 116: 2851-2859.
3. Negre-Salvayre A, Auge N, Ayala V, Basaga H, Boada J, Brenke R, Chapple S, Cohen G, Feher J, Grune T, Lengyel G, Mann GE, Pamplona R, Poli G, Portero-Otin M, Riahi Y, Salvayre R, Sasson S, Serrano J, Shamni O, Siems W, Siow RC, Wiswedel I, Zarkovic K, Zarkovic N. Pathological aspects of lipid peroxidation. Free Radic Res 2010; 44: 1125-1171.

4. Williams KJ, Tabas I. Lipoprotein retention--and clues for atheroma regression. Arterioscler Thromb Vasc Biol 2005; 25: 1536-1540.

5. Griendling KK. Novel NAD (P) H oxidases in the cardiovascular system. Heart 2004; 90: 491-493.

6. Griendling KK. ATVB in focus: redox mechanisms in blood vessels. Arterioscler Thromb Vasc Biol 2005; 25: 272-273.

7. Lyle AN, Griendling KK. Modulation of vascular smooth muscle signaling by reactive oxygen species. Physiology (Bethesda) 2006; 21 : 269-280.

8. Edirisinghe I, Arunachalam G, Wong C, Yao H, Rahman A, Phipps RP, Jin ZG, Rahman I. Cigarette-smoke-induced oxidative/ nitrosative stress impairs VEGF- and fluid-shear-stress-mediated signaling in endothelial cells. Antioxid Redox Signal 2010; 12: 1355-1369.

9. Edirisinghe I, Yang SR, Yao H, Rajendrasozhan S, Caito S, Adenuga D, Wong C, Rahman A, Phipps RP, Jin ZG, Rahman I. VEGFR-2 inhibition augments cigarette smoke-induced oxidative stress and inflammatory responses leading to endothelial dysfunction. FASEB J 2008; 22 : $2297-2310$.

10. Corretti MC, Anderson TJ, Benjamin EJ, Celermajer D, Charbonneau F, Creager MA, Deanfield J, Drexler H, Gerhard-Herman M, Herrington D, Vallance P, Vita J, Vogel R; International Brachial Artery Reactivity Task Force. Guidelines for the ultrasound assessment of endothelial-dependent flow-mediated vasodilation of the brachial artery: a report of the International Brachial Artery Reactivity Task Force. J Am Coll Cardiol 2002; 39: 257-265.

11. Yagi K. Assay for blood plasma or serum. Methods Enzymol 1984; 105: 328-331.

12. Erel O. A new automated colorimetric method for measuring total oxidant status. Clin Biochem 2005; 38: 1103-1111.

13. Erel O. A novel automated method to measure total antioxidant response against potent free radical reactions. Clin Biochem 2004; 37 : $112-119$.

14. Demirbag R, Gur M, Yilmaz R, Kunt AS, Erel O, Andac MH. Influence of oxidative stress on the development of collateral circulation in total coronary occlusions. Int J Cardiol 2007; 116: 14-19.

15. Simova I, Katova T, Denchev S, Dimitrov N. Flow-mediated dilatation has an additive value to stress ECG for the diagnosis of angiographically significant coronary atherosclerosis. J Am Soc Hypertens 2010; 4: 203-208.

16. Yoshida T, Kawano H, Miyamoto S, Motoyama T, Fukushima H, Hirai N, Ogawa H. Prognostic value of flow-mediated dilation of the brachial artery in patients with cardiovascular disease. Intern Med 2006; 5: 575-579.

17. Kitta Y, Nakamura T, Kodama Y, Takano H, Umetani K, Fujioka D, Saito Y, Kawabata K, Obata JE, Ichigi Y, Mende A, Kugiyama K. Endothelial vasomotor dysfunction in the brachial artery is associated with late in-stent coronary restenosis. J Am Coll Cardiol 2005; 46: 648-655.

18. Gokce N, Keaney JF Jr, Hunter LM, Watkins MT, Menzoian JO, Vita JA. Risk stratification for postoperative cardiovascular events vian- 
oninvasive assessment of endothelial function: a prospective study. Circulation 2002; 105: 1567-1572.

19. Guazzi M, Reina G, Gripari P, Tumminello G, Vicenzi M, Arena R. Prognostic value of flow-mediated dilatation following myocardial infarction. Int J Cardiol 2009; 132: 45-50.

20. Korkmaz H, Onalan O. Evaluation of endothelial dysfunction: flowmediated dilation. Endothelium 2008;15 (4): 157-163.

21. Neunteufl T, Heher S, Katzenschlager R, Wölfl G, Kostner K, Maurer G, Weidinger F. Late prognostic value of flow-mediated dilation in the brachial artery of patients with chest pain. Am J Cardiol 2000; 86: 207-210.

22. Perticone F, Ceravolo R, Pujia A, Ventura G, Iacopino S, Scozzafava A, Ferraro A, Chello M, Mastroroberto P, Verdecchia P, Schillaci G. Prognostic significance of endothelial dysfunction in hypertensive patients. Circulation 2001; 104: 191-196.

23. Ozaki K, Hori T, Ishibashi T, Nishio M, Aizawa Y. Effects of chronic cigarette smoking on endothelial function in young men. J Cardiol 2010; 56: $307-313$.

24. Johnson HM, Gossett LK, Piper ME, Aeschlimann SE, Korcarz CE, Baker TB, Fiore MC, Stein JH. Effects of smoking and smoking cessation on endothelial function: 1-year outcomes from a randomized clinical trial. J Am Coll Cardiol 2010; 55: 1988-1995.
25. Tarcin O, Yavuz DG, Ozben B, Telli A, Ogunc AV, Yuksel M, Toprak A, Yazici D, Sancak S, Deyneli O, Akalin S. Effect of vitamin D deficiency and replacement on endothelial function in asymptomatic subjects. J Clin Endocrinol Metab 2009; 94: 4023-4030.

26. Su Y, Liu XM, Sun YM, Jin HB, Fu R, Wang YY, Wu Y, Luan Y. The relationship between endothelial dysfunction and oxidative stress in diabetes and prediabetes. Int J Clin Pract 2008; 62: 877-882.

27. Ghiadoni L, Cupisti A, Huang Y, Mattei P, Cardinal H, Favilla S, Rindi P, Barsotti G, Taddei S, Salvetti A. Endothelial dysfunction and oxidative stress in chronic renal failure. J Nephrol 2004; 17: 512-519.

28. Cangemi R, Angelico F, Loffredo L, Del Ben M, Pignatelli P, Martini A, Violi F. Oxidative stress-mediated arterial dysfunction in patients with metabolic syndrome: Effect of ascorbic acid. Free Radic Biol Med 2007; 43: 853-859.

29. Raij L, DeMaster EG, Jaimes EA. Cigarette smoke-induced endothelium dysfunction: role of superoxide anion. J Hypertens 2001; 19: 891-897

30. Kato T, Inoue T, Morooka T, Yoshimoto N, Node K. Short-term passive smoking causes endothelial dysfunction via oxidative stress in nonsmokers. Can J Physiol Pharmacol 2006; 84: 523-529.

Received December 31, 2011. Accepted August 18, 2013. 